\author{
Maximilian C. SCHIELEIN ${ }^{1,2}$ \\ Linda TIZEK ${ }^{1,2}$ \\ Lisanne KNOBLOCH ${ }^{3}$ \\ Dirk MAABEN ${ }^{4}$ \\ Tilo BIEDERMANN ${ }^{1}$ \\ Alexander ZINK ${ }^{1}$ \\ ${ }^{1}$ Technical University of Munich, School of \\ Medicine, Department of Dermatology and \\ Allergy, Munich, Germany \\ ${ }^{2}$ Department of Medical Informatics, \\ Biometry and Epidemiology (IBE), \\ Ludwig-Maximilians-University, Munich, \\ Germany \\ ${ }^{3}$ Benedictus Hospital, Department of \\ Neurology, Tutzing, Germany \\ ${ }^{4}$ Psoriasis Pratice Network South-West, \\ Maxdorf, Germany
}

Reprints: Alexander Zink

<alexander.zink@tum.de>

Article accepted on 14/01/2021

\section{Psoriasis and addiction: assessing mental health based on a cross-sectional study in Germany}

\begin{abstract}
Background: Psoriasis is a chronic skin disease with a high mental burden. Well-known comorbidities include depression, anxiety, as well as alcohol and tobacco addiction, however, there is barely any evidence on other addictions. Objectives: The aim of this study was to estimate the prevalence of the six most common addictions among psoriasis patients in Germany and to determine associated clinical factors. Materials \& Methods: Dermatologists working in four dermatological clinics and 32 practices across Germany recruited patients between September 2018 and November 2019. This cross-sectional study contained questionnaires on six addictions, depression, anxiety, and the Dermatology Life Quality Index (DLQI). In addition, scores for the Psoriasis Area and Severity Index (PASI) were obtained by physicians. Results: Overall, 502 patients (43.4\% women; mean age: $49.7 \pm 14.6$ years) were included. Positive addictions were found in $30.3 \%$ for daily smoking, $8.6 \%$ for alcohol, $1.2 \%$ for gambling, $3.8 \%$ for internet use, $3.6 \%$ for food, and $6.0 \%$ for drugs. Younger age was associated with a higher probability of addiction except for alcohol dependency. The PASI was only significantly associated with smoking. Conclusion: Addictions seem to be common among psoriasis patients. Further research should include comprehensive data and control groups, furthermore, standardised screenings and early referrals could represent first steps to improve people-centred healthcare for patients with psoriasis.
\end{abstract}

Key words: addiction medicine, comorbidity, epidemiology, mental health, psoriasis
$\mathrm{P}$

soriasis is a chronic inflammatory systemic skin disease associated with typical cutaneous manifestations, affecting $0.5-11.4 \%$ of the global population and $2.1 \%$ of the German population [1]. It is often accompanied by comorbidities such as cardiovascular, rheumatoid, and mental diseases [2]. Mental diseases are particularly significant in psoriasis, as psoriasis lesions often affect visible body areas or the genital area, causing stigmatisation $[3,4]$ and impairment of patients' quality of life, happiness, and sex life [5-7]. Comorbid mental diseases include depression, anxiety, suicidality, and various addictions [813]. Addictions such as alcohol consumption and smoking are commonly investigated $[10,11]$, whereas data on other addictions such as gambling and drug abuse are rare [12]. To improve patients' quality of life, early detection of these comorbidities is essential. However, comorbidities often remain unrecognised [14-16]. Consequently, there is a great need for improvement to reach the goals outlined in the 'Global report on psoriasis' issued by the World Health Organisation (WHO) in 2016 [17]. This report emphasises the need for people-centred care and multidisciplinary approaches in order to minimise the burden that individuals suffer due to psoriasis. When psoriasis is treated sufficiently, a reduction of disease severity has been shown to be associated with an improvement in quality of life and decrease in depressive symptoms [8, 18-20]. Since patientphysician time is limited and limited information on risk factors for mental comorbidities is available, screening for mental comorbidities is advocated in the literature [20,21]. While some clinical risk factors for depression and anxiety are available [20-23], currently there is no evidence on risk factors for addictions.

Therefore, the aim of this study was to estimate the prevalence of the six most common addictions in Germany in patients with psoriasis and to determine clinical factors associated with the occurrence of each addiction, respectively.

\section{Materials and methods}

\section{Study population}

For this cross-sectional, paper-based, non-interventional study, participants were recruited from 32 dermatological practices and four dermatological clinics across Germany between September 2018 and November 2019. Dermatologists were invited using data from the German psoriasis physician network 'Psoriasis Praxisnetz Süd-West e.V.'. 
If physicians agreed to participate, they received material to include up to 10 patients. Additional patient sets for inclusion were provided on demand. For study participation, patients had to fulfil the following inclusion criteria: age $\geq 18$ years, diagnosed with psoriasis, able to provide written informed consent, and able to answer a German questionnaire. To minimise selection bias, dermatologists were asked to include either (1) the first 10 psoriasis patients willing to participate after the start of recruitment or (2) patients who visited within the last two months retrospectively and consecutively. Informed consent was obtained from every participant prior to inclusion. The study and all procedures were reviewed and approved by the local ethics committee of the medical faculty of the Technical University of Munich (Reference 485/17 S).

\section{Questionnaire}

Participants received a questionnaire consisting of the German versions of nine well-established questionnaires for the assessment of mental health status. A detailed description of each instrument can be found in the supplementary material and table 1. Dermatologists additionally documented patient characteristics including age, sex, body mass index (BMI), and disease severity measured by the Psoriasis Area and Severity Index (PASI).

\section{Statistics and data management}

Study size was limited by a patient recruitment time of one year in order to reduce potential seasonal influences. Descriptive data were generated for general patient characteristics and prevalence of mental disorders. Mental health outcomes for prevalence estimation and further analyses were defined by thresholds listed in table 1 . To test differences between sex, t-test, chi-squared test, or Fisher's exact test were used. Furthermore, logistic regression models were calculated to determine personal and clinical factors (age, sex, BMI, Dermatology Life Quality Index (DLQI), PASI, and positive screening for depression and anxiety) that were associated with the occurrence of one of the six addictions. Variables were added to multivariate regression models using backward stepwise selection to minimise multicollinearity. Odds ratios (OR) and adjusted OR (aOR) were calculated with the respective $95 \%$ confidence intervals (CI). Global alpha was set at 0.05 . To improve the quality of the regression models, multiple imputations were conducted. Therefore, a fully conditional specification method was used to impute missing data. In total, five imputations were generated to calculate missing data $(n=193 ; 3.0 \%)$. Afterwards, a sensitivity analysis was performed to compare the results of the multiple imputation analysis with those of the complete cases (i.e., only cases without any missing data; supplementary table 1). All data were entered twice and compared in order to detect discrepancies. In case of error, source data were accessed and datasets were corrected. IBM SPSS Statistics (Version 25, IBM Corporation, Armonk, NY, USA) was used for all analyses.

\section{Results}

\section{Patient characteristics}

A total of 502 participants were included in this study. The mean age was 49.7 years $( \pm 14.6$ years) and $218(43.4 \%)$ were women. The mean BMI was $28.1( \pm 5.4)$ and the mean PASI was $7.3( \pm 7.6)$. Mean DLQI was $7.5( \pm 7.1)$, with significantly greater impairment in women than in men $(8.4 \pm 7.3$ vs. $6.8 \pm 7.0, p=0.020)$. Nearly half of all patients $(44.8 \%)$ reported no or a small effect of psoriasis on their daily life, whereas $27.1 \%$ reported a large or extremely large impact. Overall, $29.5 \%$ of individuals screened positive for depression, with $11.4 \%$ indicating major depression. Anxiety was found in $48.8 \%$ of patients, with $17.5 \%$ having signs for an anxiety disorder (figure 1). Women were more likely to be positive for depression $(35.8 \%$ vs. $24.7 \%, p=0.006)$ and anxiety disorder than men $(20.1 \%$ vs. $14.8 \% ; p<0.001)$ (table 2$)$.

\section{Prevalence of addictions}

A total of 152 participants (30.3\%) reported daily cigarette smoking, with no significant difference between women and men (33.0\% vs. $28.2 \%, p=0.216)$ (table 2$)$. The mean duration of daily smoking was 24.2 years ( \pm 13.1 years). Of those who were smoking daily, 55.3\% $(n=84)$ stated that they smoked less than a pack per day, $34.9 \%(n=53)$ one pack per day, and 9.9\% $(n=15)$ more than a pack per day. Additionally, $8.6 \%(n=43)$ of all patients screened positive for alcoholism, with a higher proportion in men than women $(11.3 \%$ vs. $5.0 \%, p=0.020)$. A sex difference was also observed regarding addiction of gambling as only men were positive for this $(2.2 \%$ of men vs. $0 \%$ of women, $p=0.039$ ). For all other addictions, no sex difference was found. Overall, 3.8\% $(n=19)$ of the patients showed pathological internet behaviour and $2.0 \%(n=10)$ had borderline to pathological internet use. Around 3.6\% $(n=18)$ of the patients screened positive for food addiction. Furthermore, $15.0 \%(n=75)$ of the participants displayed at least a low level of abusive behaviour regarding drugs, with positive screening for drug abuse in $6.0 \%(n=30)$ (figure 1). Considering all addictions, $36.9 \%(n=185)$ of patients screened positive for at least one addiction, with no significant difference in sex (women: $37.6 \%$ vs. men: $36.2 \%, p=0.200$ ). However, considering the number of addictions, women were more likely to have one addiction $(30.7 \%$ vs. $24.3 \%)$, while men were more likely to have two or more addictions $(11.9 \%$ vs. $6.9 \%, p=0.013)$ (table 2$)$.

\section{Addiction-associated factors}

With the exception of alcohol dependency, younger age was associated with a higher chance of having all addictions. Therefore, age had the strongest influence on pathological gambling (aOR after multiple imputation $\left[\mathrm{aOR}_{\mathrm{MI}}\right]=0.93$, 95\% CI: $0.87-1.00)$ and drug addiction $\left(\mathrm{aOR}_{\mathrm{MI}}=0.94\right.$, 95\% CI: 0.92-0.97) (figure 1). Additionally, PASI was associated with smoking $\left(\mathrm{aOR}_{\mathrm{MI}}=1.04,95 \%\right.$ CI: $\left.1.01-1.07\right)$. For example, a 10-point increase in PASI was associated with a $40 \%$ increase in smoking. Furthermore, depression was significantly associated with alcohol $\left(\mathrm{aOR}_{\mathrm{MI}}=2.66\right.$, 95\% CI: $1.40-5.01)$ and drug addiction $\left(\mathrm{aOR}_{\mathrm{MI}}=2.66\right.$, 


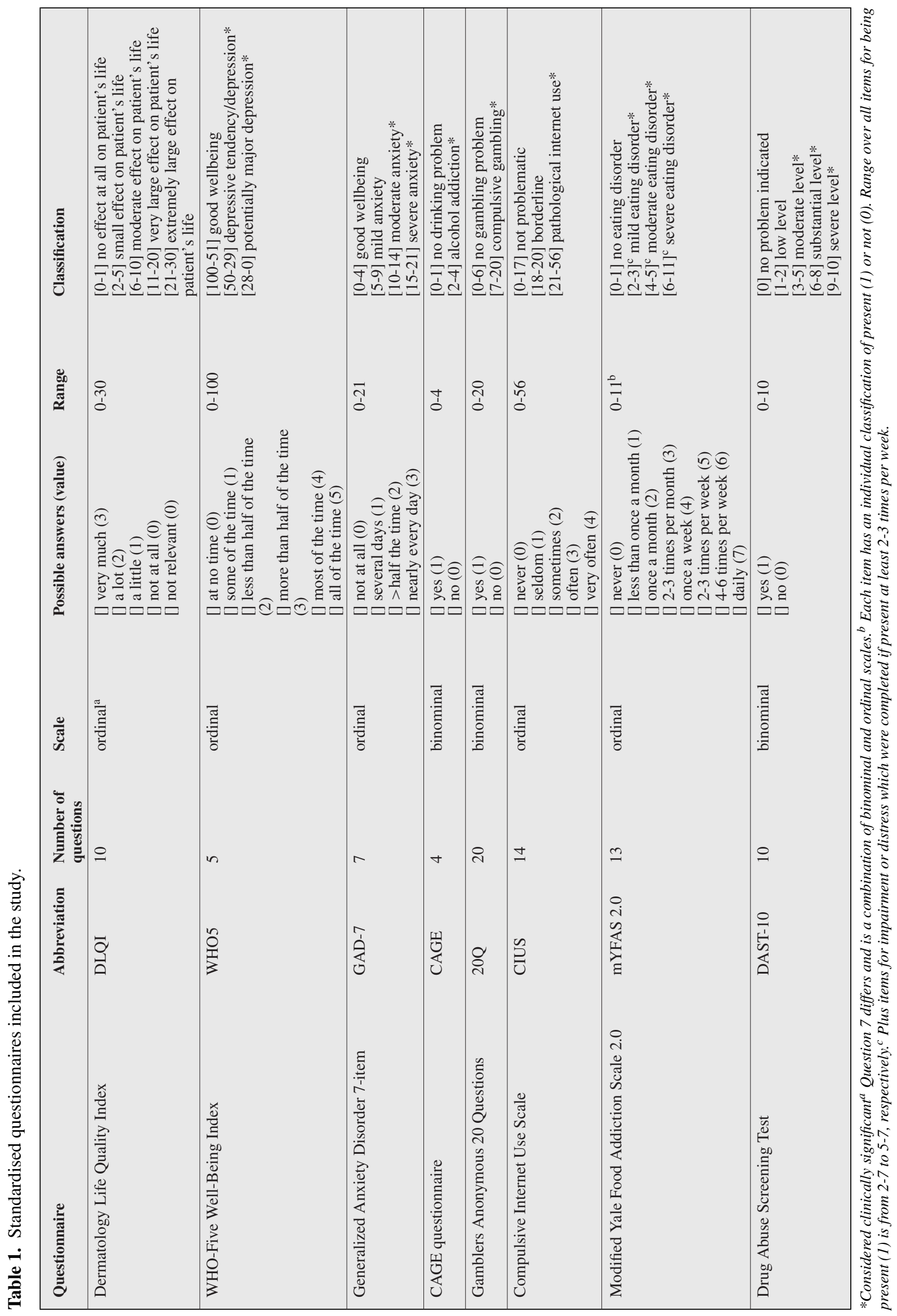




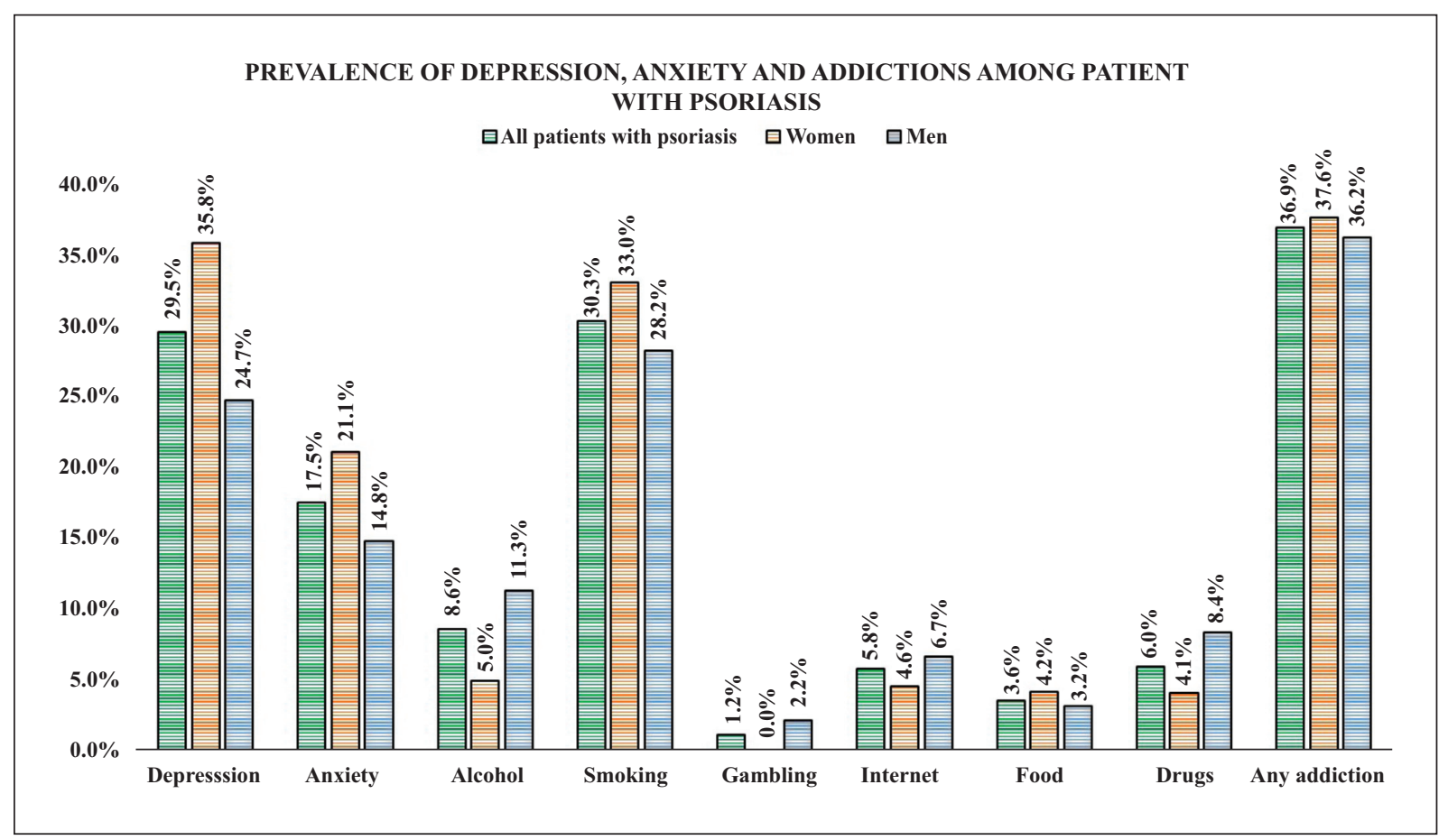

Figure 1. Prevalence of depression, anxiety and the six most common addictions in Germany (smoking, alcohol, gambling, internet use, food, and drugs), stratified by gender.

95\% CI: 1.23-5.77), whereas internet use $\left(\mathrm{aOR}_{\mathrm{MI}}=7.66\right.$, 95\% CI: 2.92-19.98) and food addiction $\left(\mathrm{aOR}_{\mathrm{MI}}=3.03\right.$, 95\% CI: 1.10-8.36) were more common in patients who had an anxiety disorder (figure 2, supplementary table 1).

\section{Discussion}

The aim of this study was to provide evidence on the estimated prevalence of six addictions in patients with psoriasis as well as to identify associated clinical factors. Overall, mental burden was high in patients with psoriasis and there was a high prevalence of addiction with nearly half of the patients reporting signs of at least one addiction. In particular, internet addiction was surprisingly high in the study population. Younger age was associated with a higher chance of most addictions.

\section{Comparison with the general population}

The study indicates that psoriasis patients have a greater likelihood of several addictions compared to representative samples of the German general population, including daily smoking (30.3\% vs. $15.1 \%)$ [24], alcohol dependency (8.6\% vs. $3.1 \%)$ [24], pathological gambling $(1.2 \%$ vs. $0.2 \%$ ) [25], and legal or illegal drug abuse (6.0\% vs. $3.2 \%-5.2 \%$ ) [24]. The increased mental burden due to skin diseases and social withdrawal might play important roles in this association [13, 26]. Despite the higher BMI in this sample compared to the general population (28.1 vs. 26.7), food addiction was found to be less prevalent among patients with psoriasis (3.6\% vs. $7.9 \%)$ [27], although the logistic regression revealed a positive association with BMI.
This difference is therefore unlikely to be related to excessive eating but may be related to a social desirability bias. Internet addiction is reported to decrease with increasing age [28, 29]. However, pathological internet use was higher in this study (3.8\%) with a mean age of 49.7 years, compared to a study including a representative sample of German adolescents with a mean age of 14.9 years $(3.2 \%)$ [30]. Another study performed by Rumpf et al. assessed internet addiction among more than 8,130 German individuals, who had a mean age of 39.9 years and used the internet regularly [28]. When adjusted to the cut-off used by Rumpf et al., the prevalence of internet addiction was equal (1.5\%), although this study population was older. Thus, the study may indicate an association between internet addiction and psoriasis, however, further investigation is required.

\section{Comparison with other studies of individuals with psoriasis}

The proportion of psoriasis patients that currently smoke varies widely in the literature, ranging from $14.0 \%$ to $51.3 \%$ [10]. Although the prevalence detected in this study was within this range, it was lower compared to that reported in another German study among psoriasis patients $(30.8 \%$ vs. $41.0 \%$ ) [12]. In a systematic review, the prevalence of alcohol addiction was reported to be between $11.1 \%$ and $28.0 \%$ in psoriasis patients [11], which is higher than that reported in this study. Studies using the same questionnaire tool as that used in this study included 60 and 102 patients with a prevalence of $18.3 \%$ and $13.5 \%$, respectively $[12,31]$. Although the prevalence of alcohol addiction was lower in this study compared to most previous studies, the prevalence exceeds the rate within the general German population (3.1\% [24]). 
Table 2. General characteristics of participants stratified by sex. Differences were compared using the unpaired t-test and Chi square test.

\begin{tabular}{|c|c|c|c|c|c|}
\hline & & $\begin{array}{l}\text { Total } \\
(n=502)\end{array}$ & $\begin{array}{l}\text { Women } \\
(n=218)\end{array}$ & $\begin{array}{l}\text { Men } \\
(n=284)\end{array}$ & $p$ value \\
\hline Age $[$ Mean \pm SD] & & $49.7 \pm 14.6$ & $50.7 \pm 14.8$ & $48.9 \pm 14.4$ & 0.164 \\
\hline BMI $[$ Mean \pm SD] & & $28.1 \pm 5.4$ & $28.3 \pm 6.5$ & $28.0 \pm 4.3$ & 0.594 \\
\hline PASI $[$ Mean \pm SD] & & $7.3 \pm 7.6$ & $6.7 \pm 6.8$ & $7.8 \pm 8.1$ & 0.098 \\
\hline DLQI [Mean \pm SD] & & $7.5 \pm 7.1$ & $8.4 \pm 7.3$ & $6.8 \pm 7.0$ & 0.020 \\
\hline \multirow{4}{*}{ Depression [n (\%)] } & No & $351(69.9)$ & $139(63.8)$ & $212(75.2)$ & \multirow{4}{*}{0.006} \\
\hline & General & $91(18.1)$ & $43(19.7)$ & $48(17.0)$ & \\
\hline & Major & $57(11.4)$ & $35(16.1)$ & $22(7.7)$ & \\
\hline & Missing & $3(0.6)$ & $1(0.5)$ & $2(0.7)$ & \\
\hline \multirow{5}{*}{ Anxiety $[n(\%)]$} & No & $248(49.4)$ & $84(38.5)$ & $164(57.7)$ & \multirow{5}{*}{$\stackrel{<}{0.001}$} \\
\hline & Mild & $157(31.3)$ & $83(38.1)$ & $74(26.1)$ & \\
\hline & Moderate & $68(13.5)$ & $36(16.5)$ & $32(11.3)$ & \\
\hline & Severe & $20(4.0)$ & $10(4.6)$ & $10(3.5)$ & \\
\hline & Missing & $9(1.8)$ & $5(2.3)$ & $4(1.4)$ & \\
\hline \multirow[t]{3}{*}{ Daily smoking [n(\%)] } & Yes & $152(30.3)$ & $72(33.0)$ & $80(28.2)$ & \multirow[t]{3}{*}{0.216} \\
\hline & No & $343(68.3)$ & $142(65.1)$ & $201(70.8)$ & \\
\hline & Missing & $7(1.4)$ & $4(1.8)$ & $3(1.1)$ & \\
\hline $\begin{array}{l}\text { Years of smoking } \\
{[\text { Mean } \pm \text { SD] }}\end{array}$ & & $24.2 \pm 13.1$ & $25.3 \pm 13.3$ & $23.3 \pm 12.9$ & 0.355 \\
\hline \multirow{3}{*}{ Alcohol $[n(\%)]$} & Yes & 43 (8.6) & $11(5.0)$ & $32(11.3)$ & \multirow[t]{3}{*}{0.020} \\
\hline & No & $444(88.4)$ & $195(89.4)$ & $249(87.7)$ & \\
\hline & Missing & $15(3.0)$ & $12(5.5)$ & $3(1.1)$ & \\
\hline \multirow[t]{3}{*}{ Gambling $[n(\%)]$} & Yes & $6(1.2)$ & $0(0.0)$ & $6(2.1)$ & \multirow{3}{*}{$0.039 *$} \\
\hline & No & $481(95.8)$ & $210(96.3)$ & $271(95.4)$ & \\
\hline & Missing & $15(3.0)$ & $8(3.7)$ & $7(2.5)$ & \\
\hline \multirow{4}{*}{ Internet use [n(\%)] } & No & 449 (89.4) & $195(89.4)$ & $254(89.4)$ & \multirow{4}{*}{0.583} \\
\hline & Borderline & $10(2.0)$ & $4(1.8)$ & $6(2.1)$ & \\
\hline & Pathological & $19(3.8)$ & $6(2.8)$ & $13(4.6)$ & \\
\hline & Missing & $24(4.8)$ & $13(6.0)$ & $11(3.9)$ & \\
\hline \multirow{5}{*}{ Food $[n(\%)]$} & No & 475 (94.6) & $205(94.0)$ & $270(95.1)$ & \multirow{5}{*}{0.423} \\
\hline & Mild & $7(1.4)$ & $5(2.3)$ & $2(0.7)$ & \\
\hline & Moderate & $4(0.8)$ & $1(0.5)$ & $3(1.1)$ & \\
\hline & Severe & $7(1.4)$ & $3(1.4)$ & $4(1.4)$ & \\
\hline & Missing & $9(1.8)$ & $4(1.8)$ & $5(1.8)$ & \\
\hline \multirow{6}{*}{ Drugs [n (\%)] } & No & 406 (80.9) & $174(79.8)$ & $232(81.7)$ & \multirow{6}{*}{0.484} \\
\hline & Low level & $45(9.0)$ & $22(10.1)$ & $23(8.1)$ & \\
\hline & Moderate level & $18(3.6)$ & $5(2.3)$ & $13(4.6)$ & \\
\hline & Severe level & $8(1.6)$ & $2(0.9)$ & $6(2.1)$ & \\
\hline & Substantial level & $4(0.8)$ & $2(0.9)$ & $2(0.7)$ & \\
\hline & Missing & $21(4.2)$ & $13(6.0)$ & $8(2.8)$ & \\
\hline
\end{tabular}


Table 2. (Continued).

\begin{tabular}{|lllll|}
\hline & $\begin{array}{l}\text { Total } \\
(\boldsymbol{n = 5 0 2})\end{array}$ & $\begin{array}{l}\text { Women } \\
(\boldsymbol{n = 2 1 8})\end{array}$ & $\begin{array}{l}\text { Men } \\
(\boldsymbol{n}=\mathbf{2 8 4})\end{array}$ & $\begin{array}{c}\boldsymbol{p} \text { value } \\
\text { Addictions }[\boldsymbol{n}(\boldsymbol{\%})]\end{array}$ \\
\cline { 2 - 5 } & None & $250(49.8)$ & $95(43.6)$ & $69(24.3)$ \\
\hline & $136(27.1)$ & $67(30.7)$ & $22(7.7)$ \\
\hline The & $36(7.2)$ & $14(6.4)$ & $12(4.2)$ \\
\hline
\end{tabular}

*Fisher's exact test was used as the requirements for the Chi square test were not fulfilled.SD: standard deviation; BMI: body mass- index; DLQI: Dermatology Life Quality Index; PASI: Psoriasis Area and Severity Index. Significant results are presented in bold.

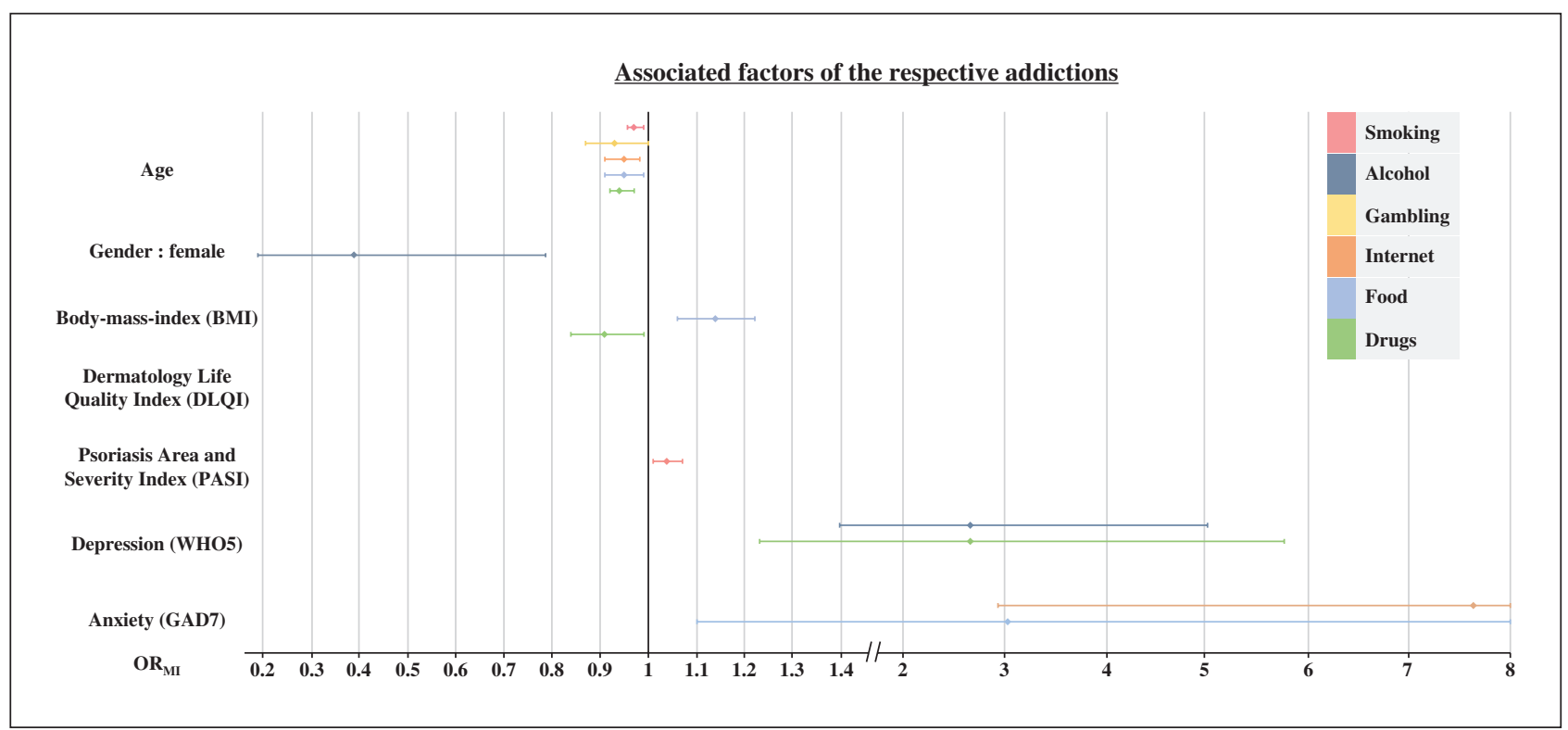

Figure 2. Personal and clinical factors associated with each addiction.

BMI: body-mass index; DLQI: Dermatology Life Quality Index; PASI: Psoriasis Area and Severity Index; WHO5: WHO-Five Well-Being Index; GAD-7: Generalized Anxiety Disorder 7-item.

The prevalence of internet addiction was lower compared to another study including individuals with psoriasis who were reached via online self-help groups $(8.5 \%)[26]$. As the prevalence is reported to be higher in people with psoriasis compared to patients with psoriasis, the results underline the importance of a people-centred care, as demanded by the WHO [17].

When considering the other addictions examined, data are limited to one study including 102 patients from a single university hospital [12]. The authors found higher rates for compulsive gambling $(2.0 \%$ vs. $1.2 \%)$, but a lower rate for food $(3.1 \%$ vs. $3.6 \%)$ and drug addiction (1.0\% vs. 2.4\%; threshold adapted to Zink et al.) compared to this sample [12]. As there were no considerable differences regarding age and severity of psoriasis, the different findings emphasise the need for further clarification and research to potentially include data extracted from health insurance registries.

\section{Addiction-associated factors}

The individual and clinical factors associated with all six addictions have not been previously investigated. The multiple regression models reveal that for most of the addictions, younger age had a significant influence. Although the discussion on age and addiction within the current literature is highly controversial $[32,33]$, what stands out for all addictions, expect alcohol addiction, is that the chance of having an addiction significantly decreases with increasing age. Based on backward selection, only daily smoking was associated with a higher PASI. This might either indicate the absence of a classic dose-response relationship or that conventional strategies to classify disease severity in a cross-sectional manner might not comprehensively reflect the individual burden experienced by patients [34]. Furthermore, all addictions apart from smoking and gambling were associated with depression or anxiety, which is in accordance with the literature [35]. These findings strengthen the 
reliability of the study results. However, no specific clinical factor can be identified as a potential predictor even though their prevalence was higher than in the general population. Therefore, the findings also emphasise the need for further research and standardized screening.

\section{Strengths and limitations}

A major strength of this study is the strict set of criteria for consecutive inclusion of patients that was applied to reduce selection bias. Additionally, recruitment was carried out in 36 medical settings throughout Germany. There are some study limitations, however. First, there is a potential for data and selection bias as participation for dermatologists was voluntary and this can lead to a self-selected subgroup of dermatologists. Second, screening for addictions was performed using standardised questionnaires without a corresponding control group, thus comparisons are only indirect. Although the questionnaires are validated and all showed acceptable results for specificity and sensitivity [3], it should be taken into account that these findings do not represent diagnoses but rather an estimate of the respective outcomes, and provide no valid information on severity of the respective addictions. Third, a certain degree of social and desirability bias should be considered. Patients are not always willing to disclose information regarding sensitive and potentially stigmatising data [36, 37]. Furthermore, no duration or time frame for psoriasis or addiction was documented, therefore the direct or indirect nature of the relationship between the two cannot be addressed.

\section{Conclusion}

In conclusion, the prevalence of addictions, especially internet addiction, was high among patients with psoriasis. To further verify the association between the prevalence of addiction and psoriasis, future research should also include a suitable control group or data from health insurance registries. The study findings underline the importance of implementing a routine, relatively rapid assessment of psychological comorbidities when assessing the severity of psoriasis, which could be easily implemented in psoriasis care [21-23]. As different clinical variables were associated with different addictions, a possible first step could include routine and standardised screening as well as referral for early detection and treatment of these psychological disturbances in order to improve the quality of people-centred care.

Disclosures. Financial support: Parts of this study were supported by an unrestricted research grant by Novartis Pharma GmbH. Conflicts of interest: MCS received speaker's honoraria and/or was financially supported for the presentation of scientific posters by Janssen-Cilag, Novartis. LT was financially supported for the presentation of scientific posters by Novartis Pharma GmbH. LK has no conflicts of interest to declare. DM has served as a consultant and/or paid speaker for and/or has received honoraria for consulting and/or obtained reimbursed travel expenses and/or participated in clinical trials sponsored by companies that manufacture drugs used for the treatment of psoriasis including AbbVie, Almirall, Amgen, Biogen (Biogen Idec), Celgene, Eli Lilly, Janssen-Cilag, Leo, Medac, MSD (formerly Essex, Schering-Plough), Mundipharma, Novartis, Pfizer (formerly Wyeth), USB. TB provided advice for, or obtained an honorarium for talks or research grants from the following companies: Celgene, Novartis, Lilly. AZ has been an advisor and/or received speaker's honoraria and/or received grants and/or participated in clinical trials for the following companies: AbbVie, Almirall, Celgene, Eli Lilly, Janssen-Cilag, Novartis.

\section{Supplementary data}

Supplementary data associated with this article can be found, in the online version, at doi:10.1684/ejd.2021.4146. Table S1: Comparison between complete-case analysis and multiple imputation analysis for clinical factors associated with depression, anxiety or addictions.

\section{References}

1. Michalek IM, Loring B, John SM. A systematic review of worldwide epidemiology of psoriasis. J Eur Acad Dermatol Venereol 2017; 31:205-12.

2. Takeshita J, Grewal S, Langan $S M$, et al. Psoriasis and comorbid diseases: epidemiology. J Am Acad Dermatol 2017; 76: 377-90.

3. Topp J, Andrees V, Weinberger NA, et al. Strategies to reduce stigma related to visible chronic skin diseases: a systematic review. J Eur Acad Dermatol Venereol 2019; 33: 2029-38.

4. Pearl RL, Wan MT, Takeshita J, et al. Stigmatizing attitudes toward persons with psoriasis among laypersons and medical students. J Am Acad Dermatol 2019; 80: 1556-63

5. Sampogna F, Abeni D, Gieler U, et al. Impairment of sexual life in 3,485 dermatological outpatients from a multicentre study in 13 European countries. Acta Derm Venereol 2017; 97: 478-82.

6. Schielein MC, Tizek L, Schuster B, et al. Genital psoriasis and the influence on sexual distress - a people-centered cross-sectional study in Germany. Acta Derm Venereol 2020; 100: adv00151.

7. Schuster B, Ziehfreund S, Albrecht $\mathrm{H}$, et al. Happiness in dermatology: a holistic evaluation of the mental burden of skin diseases. J Eur Acad Dermatol Venereol 2020; 34: 1331 1-9.

8. Koo J, Marangell LB, Nakamura M, et al. Depression and suicidality in psoriasis: review of the literature including the cytokine theory of depression. J Eur Acad Dermatol Venereol 2017; 31 : 1999-2009.

9. Matterne U, Baumeister SE, Apfelbacher CJ. Suicidality and risk of suicidality in psoriasis: a critical appraisal of two systematic reviews and meta-analyses. Br J Dermatol 2019; 181:717-21.

10. Armstrong AW, Harskamp CT, Dhillon JS, et al. Psoriasis and smoking: a systematic review and meta-analysis. $\mathrm{Br} J$ Dermatol 2014; 170: 304-14.

11. Brenaut E, Horreau C, Pouplard C, et al. Alcohol consumption and psoriasis: a systematic literature review. J Eur Acad Dermatol Venereol $2013 ; 27: 30-5$

12. Zink $A$, Herrmann $M$, Fischer $T$, et al. Addiction: an underestimated problem in psoriasis health care. J Eur Acad Dermatol Venereol 2017; 31: 1308-15.

13. Egeberg A, Thyssen JP, Wu JJ, et al. Risk of first-time and recurrent depression in patients with psoriasis: a population-based cohort study. Br J Dermatol 2019; 180: 116-21. 
14. Dauden E, Blasco AJ, Bonanad C, et al. Position statement for the management of comorbidities in psoriasis. J Eur Acad Dermatol Venereol 2018; 32: 2058-73.

15. Strohal R, Kirby B, Puig L, et al. Psoriasis beyond the skin: an expert group consensus on the management of psoriatic arthritis and common co-morbidities in patients with moderate-to-severe psoriasis. $J$ Eur Acad Dermatol Venereol 2014; 28: 1661-9.

16. Radtke MA, Mrowietz U, Feuerhahn J, et al. Early detection of comorbidity in psoriasis: recommendations of the National Conference on Healthcare in Psoriasis. J Dtsch Dermatol Ges 2015; 13: 674-90.

17. Michalek IM, Loring $B$, John SM. Global report on psoriasis. Geneva, Switzerland: World Health Organization, 2016.

18. Lamb RC, Matcham F, Turner MA, et al. Screening for anxiety and depression in people with psoriasis: a cross-sectional study in a tertiary referral setting. Br J Dermatol 2017; 176: 1028-34.

19. Papp KA, Reich K, Paul C, et al. A prospective phase III, randomized, double-blind, placebo-controlled study of brodalumab in patients with moderate-to-severe plaque psoriasis. $\mathrm{Br} J$ Dermatol 2016; 175: 273-86.

20. Nicholas MN, Gooderham M. Psoriasis, depression and suicidality. Skin Therapy Lett 2017; 22: 1-4.

21. Lesner K, Reich A, Szepietowski JC, et al. Determinants of psychosocial health in psoriatic patients: a multi-national study. Acta Derm Venereol 2017; 97: 1182-8.

22. Kimball $A B$, Jacobson $C$, Weiss $S$, et al. The psychosocial burden of psoriasis. Am J Clin Dermatol 2005; 6: 383-92.

23. Scharloo $M$, Kaptein AA, Weinman J, et al. Patients' illness perceptions and coping as predictors of functional status in psoriasis: a 1-year follow-up. Br J Dermatol 2000; 142: 899-907.

24. Atzendorf J, Rauschert C, Seitz N-N, et al. The use of alcohol, tobacco, illegal drugs and medicines. Dtsch Arztebl Int 2019; 116:577-84.

25. Erbas $B$, Buchner UG. Pathological gambling: prevalence, diagnosis, comorbidity and intervention in Germany. Dtsch Arztebl Int 2012; 109: 173-9.
26. Schielein MC, Tizek L, Schuster B, et al. Always online? Internet addiction and social impairment in psoriasis across Germany. J Clin Med 2020; 9: 1818

27. Hauck C, Weiß A, Schulte EM, et al. Prevalence of 'food addiction' as measured with the Yale Food Addiction Scale 2.0 in a representative German sample and its association with sex, age and weight categories. Obes Facts 2017; 10: 12-24.

28. Rumpf H-J, Vermulst AA, Bischof $A$, et al. Occurence of internet addiction in a general population sample: a latent class analysis. Eur Addict Res 2014; 20: 159-66.

29. Carli $V$, Durkee $T$, Wasserman $D$, et al. The association between pathological internet use and comorbid psychopathology: a systematic review. Psychopathology 2013;46: 1-13.

30. Wartberg L, Kriston L, Kammerl R, et al. Prevalence of pathological internet use in a representative German sample of adolescents: results of a latent profile analysis. Psychopathology 2015; 48: 25-30.

31. Buchsbaum DG, Buchanan RG, Centor RM, et al. Screening for alcohol abuse using CAGE scores and likelihood ratios. Ann Intern Med 1991; 115:774-7.

32. Koechl B, Unger A, Fischer G. Age-related aspects of addiction. Gerontology 2012; 58: 540-4

33. Argyriou $E$, Um M, Carron $C$, et al. Age and impulsive behavior in drug addiction: $A$ review of past research and future directions. Pharmacol Biochem Behav 2018; 164: 106-17.

34. Tizek L, Schielein MC, Zink A. PeakPASI: A new measurement tool in psoriasis care. J Am Acad Dermatol 2020; 84: 1730-2.

35. Vondráčková $P$, Gabrhelík R. Prevention of Internet addiction: A systematic review. J Behav Addict 2016; 5: 568-79.

36. Schnyder N, Panczak R, Groth N, et al. Association between mental health-related stigma and active help-seeking: systematic review and meta-analysis. Br J Psychiatry 2017; 210: 261-8.

37. Zink $A$, Schuster $B$, Rüth $M$, et al. Medical needs and major complaints related to pruritus in Germany: a 4 -year retrospective analysis using Google AdWords Keyword Planner. J Eur Acad Dermatol Venereol 2019;33: 151-6. 